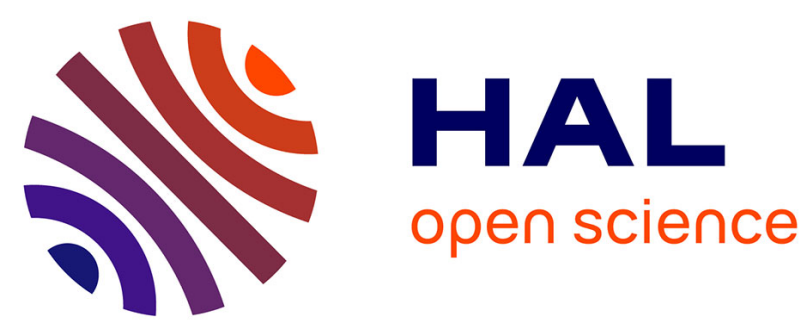

\title{
The origin of the stereoselective alkylation of 3-substituted-2-oxopiperazines: A computational investigation
}

Christine Cézard, Benjamin Bouvier, Alexandra Dassonville-Klimpt, Pascal

Sonnet

\section{To cite this version:}

Christine Cézard, Benjamin Bouvier, Alexandra Dassonville-Klimpt, Pascal Sonnet. The origin of the stereoselective alkylation of 3-substituted-2-oxopiperazines: A computational investigation. Computational and Theoretical Chemistry, 2016, 1078, pp.1-8. 10.1016/j.comptc.2015.12.005 . hal-02395301

\section{HAL Id: hal-02395301 \\ https://hal.science/hal-02395301}

Submitted on 9 Dec 2019

HAL is a multi-disciplinary open access archive for the deposit and dissemination of scientific research documents, whether they are published or not. The documents may come from teaching and research institutions in France or abroad, or from public or private research centers.
L'archive ouverte pluridisciplinaire HAL, est destinée au dépôt et à la diffusion de documents scientifiques de niveau recherche, publiés ou non, émanant des établissements d'enseignement et de recherche français ou étrangers, des laboratoires publics ou privés. 
The origin of the stereoselective alkylation of 3-substituted-2-oxopiperazines: A computational investigation Christine Cézard,* Benjamin Bouvier, Alexandra Dassonville-Klimpt and Pascal Sonnet

Laboratoire de Glycochimie, des Antimicrobiens et des Agroressources (LG2A) - CNRS FRE 3517, UFR de Pharmacie, Université de Picardie - Jules Verne, 1 Rue des Louvels, 87037 Amiens Cedex 1, France

\begin{abstract}
2-oxopiperazines and their derivatives are important pharmacophores found in numerous bioactive products. The potency of these compounds depends on the nature and/or position of their substituent(s) as well as on their chirality. Hence, it is important to develop, control and optimize synthetic routes leading to enantiomerically pure substituted 2-oxopiperazines. In this work we report on the origin of this stereoselectivity, upon alkylation of 2-oxopiperazines at position $\mathrm{C}_{3}$, studied by means of quantum chemistry calculations. Indeed, this alkylation with methyl chloride is predicted to afford mainly the exo product with a 98:2 ratio. To this purpose, we model the reaction path leading to both enantiomers by scrutinizing the structures and energetics of the pre-reaction complexes, the transition states and the postreaction complexes. The computational results are in good agreement with the experimental observations, and provide valuable insights into the origins of this specificity. From the conformational analysis of the piperazine ring and of intramolecular interaction patterns, we show that the enantiofacial discrimination is achieved by a subtle balance between sterical hindrance and control of the conformation of the piperazine ring.
\end{abstract}

* Corresponding author:

christine.cezard@u-picardie.fr

Keywords: 2-oxopiperazine, alkylation, DFT calculations, transition states, reaction path, stereospecificity 
Introduction

Piperazines, symmetrical cyclic 6-ring molecules containing four carbon atoms and two nitrogen atoms at opposing position (see Figure 1a), represent a broad class of chemical compounds with many pharmacological properties and applications. Molecules featuring a piperazine scaffold have been reported to have diverse biological activities (antihistaminic, antihelmintic, anticancer, antidepressant, antifungal, antibacterial, ...) depending on their substitution.[1-9] Indeed, piperazines can be substituted at nitrogen or carbon atoms, conferring the newly formed molecule with specific activities. For instance, simple $N$ substituted piperazines can be found in numerous drugs and/or recreational drugs.[10] While substituting a piperazine on a nitrogen atom remains rather easy, a stereo-controlled substitution on a carbon position remains quite a challenge to organic chemists. Methods used for the preparation of asymmetric substituted piperazines include enantioselective hydrogenation,[11] stereoselective alkylation of 2-oxopiperazines using a chiral auxiliary,[12-17] $\alpha$-lithiation mediated by chiral ligands such as (-)-sparteine[18, 19] or synthesis from a chiral pool of compounds such as naturally occurring amino acids.[20-23] Nowadays, because of numerous recurrent positive hits in screenings, piperazine derivatives and especially their keto analogues are considered as important and privileged scaffolds in medicinal chemistry.[24] In this paper, we will focus on 2-oxopiperazines (or piperazin-2-ones, i.e. piperazines featuring a carbonyl function at position 2, see Figure 1b) and their chiral syntheses and/or substitutions. Although less frequently employed in pharmaceutical chemistry, oxopiperazines[25] are important pharmacophores particularly found in bioactive drugs used in central nervous system therapies,[26] used to mimic inverse $\gamma$-turns in peptides,[27] or as efficient chiral ligands in enantioselective catalysis.[28] Rhodotorulic acid ( $N$-[3-[5-[3-[acetyl(hydroxy)amino]propyl]-3,6dioxopiperazin-2-yl]propyl]- $N$-hydroxyacetamide, see Figure 1c), for instance, a natural iron chelator produced by Rhodotorula pilimanae,[29] features two asymmetric centers at position 3 and 6 with identical configurations. Swapping of one of those chiralities impacts the formation (rate and spatial arrangement) of the corresponding iron complexes.[30, 31] Hence, when considering 2-oxopiperazine derivatives, it is of paramount importance to master enantioselective synthesis routes, as the presence and chirality of substituents at the different positions of the piperazine heterocycle has a direct and significant influence on the biological activity.

Our group is specialized in asymmetric substitution of 2-oxopiperazines at various C-positions and stereosynthesis of polysubstituted oxopiperazines.[32, 33] We have, in particular, proposed a new pathway to chiral 3-substituted-2-oxopiperazines based on a stereoselective alkylation of 1,4-disubstituted-2oxopiperazines with different electrophiles $\left(\mathrm{BnBr}, \mathrm{CH}_{3} \mathrm{Cl}, \ldots\right)$ by using a chiral alcohol inductor grafted to $\mathrm{N}_{1}$ (Scheme 1). While such syntheses are now routine and afford $\mathrm{C}_{3}$-1,4-disubstituted-2-oxopiperazines with high yields and diastereomeric excesses $(>96 \%)$, the mechanisms underlying this stereospecifity are still unclear. To address this shortcoming, we study the $\mathrm{C}_{3}$-alkylation of 1,4-disubstituted-piperazin-2-one (1'R)1 using quantum chemical methods. To alleviate the cost of accurate calculations on these molecules, we use methyl chloride as a model electrophile (Scheme 2). We focus on the impact of (i) reaction energetics, (ii) 
transition states structures and (iii) reaction paths on alkylation stereospecificity. Similar studies have already been performed on piperidine[34-36] and piperidone[37] derivatives but to the best of our knowledge, no such studies have ever been performed on oxopiperazine derivatives.

\section{Methods}

Models. We wish to make it clear that the species chosen as targets of our computational study may not correspond to the actual reactive intermediates present in solution and described in Scheme 1. Upon addition of a strong base such as LDA on reactant $\mathbf{1}$ in THF, deprotonation of both the hydroxyl function and position $\mathrm{C}_{3}$ will likely occur; the reactive species thus formed would present two anionic sites, which would subsequently be chelated by lithium. The hydroxyl function, being more acidic, is depronated before the $\mathrm{C}_{3}$ position and thereby binds lithium first. Micouin et al. proposed a potential mechanism involving a transition state featuring two lithium cations to explain the exo preference of the reaction.[38] Modeling the reactive species using two anionic sites or chelated by two lithium cations did not seem desirable as the incoming methyl chloride and/or the $\mathrm{Li}^{+}$would not be able to discriminate between the two reactive sites and consequently either aggregate or repulse one another. Moreover, it is known that lithium conjugates used in organic synthesis (organolithium reagents, lithium enolates, ...) form oligomers that can only achieve sufficient stabilization via interactions with other molecules that help fulfill the octet rule on lithium [39, 40] (e.g., solvents such as THF); yet the choice of the number of solvent molecules to explicitly include in quantum chemistry calculations, as well as their placement around lithium, raise difficult practicality issues. Hence, as a first step toward the understanding of the stereospecificity of the reaction, we have chosen not to introduce lithium (whether in form of single ions or aggregated with THF molecules) in our initial model, but to retain the hydroxyl group in the reactant. This simplified model provides a cost-effective approximation of the actual reactive species, considering that the hydrogen atom belongs to the first group of the periodic table. Nevertheless, it has been suggested[38, 41] that in such systems, coordination with a $\mathrm{Li}^{+}$cation may be the key factor in the stereospecificity; to ascertain whether this is the case, we performed an additional series of calculations with an actual lithium atom (in place of the hydroxyl hydrogen) bridging the two anionic sites together. The presence of the second lithium atom in the calculations is not mandatory, as it does not influence the stereoselectivity. This fact was demonstrated by a series of experiments[42] and by theoretical studies[37] on systems featuring one negatively charged oxygen.

Computational Details. All calculations were performed using the Gaussian 09 program.[43] Full geometry optimizations were performed in the gas phase with the hybrid B3LYP density functional method[44-46] using the $6-31+\mathrm{G}^{*}$ basis set, which represents a good compromise between accuracy and computational cost. Indeed, considering the large size of the systems, using higher-level methods for full optimization would not have been cost-effective. When mentioned, single point MP2/6-31+G* calculations were subsequently undertaken to determine the relative energies of the different products formed during the alkylation reactions. Frequency calculations were performed to verify the true nature of stationary points. 
Vibrational frequency calculations gave only one imaginary frequency for all transition structures, confirming their saddle-point nature, whereas all other structures were verified as minima with all-positive frequency values. Zero-point, thermal and entropic corrections at a pressure of $1 \mathrm{~atm}$ and a temperature of $195 \mathrm{~K}$ (unless otherwise specified) were added to the electronic energies to calculate the Gibbs free energies in the gas phase. Basis Superposition Set Errors (BSSE) can be neglected in calculations of such systems as discussed in references [35] and [37]. Intrinsic Reaction Coordinate analyses have been conducted to obtain the pre-reaction and post-reaction complexes connected to a transition state. The quantum theory of atoms in molecules (QTAIM) derived data were calculated with the Multiwfn software.[47]

To estimate the influence of solvation, SCRF continuum calculations were performed at the B3LYP/6$31+\mathrm{G}^{*}$ level of theory. Calculations were performed in water $(\varepsilon=78.3553)$, n-octanol $(\varepsilon=9.8629)$, tetrahydrofuran $(\varepsilon=7.4257)$, chloroform $(\varepsilon=4.7113)$ and carbontetrachloride $(\varepsilon=2.2280)$ to estimate the effect of the increasing polarity of the solvent.

\section{Results and discussion}

The stereoselectivity of the alkylation in position 3 of 2-oxopiperazine (1'R)-1 was examined. To this purpose, the reaction of the enolate derived from $\left(1^{\prime} R\right)-1$ with methyl chloride as the electrophile was investigated (see Scheme 1) in the gas phase as well as in solution. In a first step, conformational searches of the different reactants $\left(\left(1^{\prime} R\right)-1\right.$ and the enolate derived from $\left.\left(1^{\prime} R\right)-\mathbf{1}\right)$ and products $\left(\left(1^{\prime} R, 3 S\right)-2\right.$ exo and $\left(1^{\prime} R, 3 \mathrm{R}\right)-2$ endo) have been performed in the gas phase, which led to two representative families of isomers, located within a range of $10 \mathrm{kcal} . \mathrm{mol}^{-1}$ and showing different intramolecular hydrogen bonding patterns involving the hydroxyl function of the piperazine ring (see Figure 2), namely HB-1 and HB-2. HB-1 refers to the interaction between $-\mathrm{OH}$ and the carbonyl function $-\mathrm{C}=\mathrm{O}$ of the piperazine ring, whereas HB-2 refers to the interaction between- $\mathrm{OH}$ and the ether function $-\mathrm{C}-\mathrm{O}-\mathrm{C}-$ of the $-\mathrm{OBn}$ group.

While the most stable structure for $\left(1^{\prime} R\right)-1$ is based on pattern HB-2, the HB-1 pattern is found in the most stable structures for the enolate and both the products. The free energy differences G(HB-1) - G(HB-2) in the gas phase are $+0.5,-8.1,-2.0$ and -0.6 for $\left(1^{\prime} R\right)-1$, the enolate of $\left(1^{\prime} R\right)-1$, the exo product and the endo product, respectively. It has to be acknowledged that the position/direction of the OBn substituent does not alter the global stability as many conformers differing only by the OBn position lie within less than one kcal.mol-1.

Energy and free energy differences when considering the most stable structure of each compound (reactant: enolate from $\left(1^{\prime} R\right)-1$ and products $\left(1^{\prime} R, 3 S\right)-2$ and $\left.\left(1^{\prime} R, 3 R\right)-2\right)$ are reported in Table 1 . These results indicate that the $\left(\left(1^{\prime} R, 3 S\right)-2\right.$, i.e. exo $)$ product is always favored, independently of the calculation method used. B3LYP and MP2 methods provide comparable results when considering the gas phase, but the latter predicts a slighter stabilization of the exo product in solvent. Solvation free energies are of the same magnitude than that in the gas phase, indicating that stereoselectivity is unaffected by the polarity of the solvent. 
Experimentally, the $\left(1^{\prime} R, 3 S\right)-\mathbf{2}:\left(1^{\prime} R, 3 R\right)-\mathbf{2}$ ratio is at least $98: 2,[32,33]$ which implies a free energy difference of at least $1.5 \mathrm{kcal} . \mathrm{mol}^{-1}$ under experimental conditions $\left(1 \mathrm{~atm},-78^{\circ} \mathrm{C}\right)$.

In order to investigate the influence of kinetic effects in the stereoselective alkylation of (1'R)-1, the free energies of the transition states and their respective pre- and post-reaction (abbreviated as PreRC and PostRC, respectively in the remainder of the text) complexes have to be evaluated. In principle, many conformations should be considered, but as previously mentioned, conformational searches of the free enolate followed by clustering generated two main families of structures differing by the nature of their intramolecular hydrogen bond. Within these families, two sub-groups emerged according to the conformation of the piperazine ring and the position of the -OBn substituent, hence eight different enol conformations. Representative geometries of the two families with their sub-structures are represented in Figures SI1 and SI2 along with their relative energies. The transition structures for both exo and endo attacks of methyl chloride based on these eight families of conformers were located and in all cases the exo TS were more stable than the endo ones. The most stable enols for each families present an additional stabilization due to an interaction with the-OBn group. However, it is interesting to note that, depending on the intramolecular HB pattern considered, the most stable enols do not present the same piperazine cycle conformation. Hence, based on these considerations, we have selected two structural matching structures (one from each HB family) that seemed the most appropriate from a chemical point of view, featuring: (i) a "free" -OBn moiety, so as to avoid interaction with the reactive site and not to favor one attack over the other because of obvious steric hindrance upon approach of the methyl chloride and (ii) the HB-1 conformation of the piperazine cycle. Subsequently, starting from these two enols, transition structures (TS) for both exo and endo attacks of methyl chloride were located and the optimized structures of both the prereaction and post-reaction complexes were obtained from IRC calculations.

In the remainder of the text, we will focus on two reaction paths (RP):

(i) RP1 involving structures featuring the HB-1 intramolecular hydrogen bond, i.e. the interaction between the alcohol function $-\mathrm{OH}$ and the carbonyl function $-\mathrm{C}=\mathrm{O}$ of the piperazine ring (see Table 2 as well as Figures 3 and SI3-5 featuring the structures and energies of PreRC1, TS1 and PostRC1) and

(ii) RP2 involving structures featuring the HB-2 intramolecular hydrogen bond, i.e. the interaction between the alcohol function $-\mathrm{OH}$ and the ether function $-\mathrm{C}-\mathrm{O}-\mathrm{C}-$ of the $-\mathrm{OBn}$ group (see Table 3 as well as Figures 4 and SI6-8 representing the structures and energies of PreRC2, TS2 and PostRC2).

Pre-Reaction Complexes (PreRC). First of all, it has to be acknowledged that the energy difference between the exo structures of PreRC1 and PreRC2 is in favor of PreRC1 by $5.6 \mathrm{kcal}^{\mathrm{mol}}{ }^{-1}$. Depending on the intramolecular hydrogen bonding pattern considered, the pre-reaction complexes leading to the endo 
$\left(1^{\prime} R, 3 R\right)-2$ and exo $\left(1^{\prime} R, 3 S\right)-2$ products do not present similar free energy stabilities. While the two conformations show similar energies for HB-2 with a methyl chloride adopting a quite symmetrical position for both attacks, a difference of $1.4 \mathrm{kcal}^{\mathrm{mol}}{ }^{-1}$ is observed for HB-1 in favor of the exo approach. Indeed the position of the $\mathrm{OH}^{\cdots} \mathrm{O}$ hydrogen bond influences the endo ingress of the methyl chloride. Although the $\mathrm{C}_{\mathrm{M}^{-}}$ $\mathrm{C}_{3}$ distance is shorter for the endo approach (3.51 $\AA$ vs. $\left.3.62 \AA\right)$, the $\mathrm{C}-\mathrm{H} \cdots \mathrm{O}$ interaction between the carbonyl oxygen and one of the hydrogen atoms of the methyl chloride is much weaker (3.86 $\AA$ s. $2.17 \AA$ ). Such a short distance is indicative of the existence of a bond path linking the two atoms, which was indeed confirmed in the exo complex with an electron density $\rho_{\mathrm{bcp}}=0.0181$ au at bond critical point. No such path was identified in the endo complex. These interactions and values are in agreement with those of Soteras et al. while studying the alkylation of oxazolopiperidones.[37] In RP2, by construction, the incoming of the methyl chloride is not impeded by the presence of the intramolecular hydrogen bond, hence the similar positions of the methyl chloride and the similar energies. The $\mathrm{C}-\mathrm{H} \cdots \mathrm{O}$ distances between the carbonyl oxygen and one of the hydrogen atoms of the methyl chloride are $2.13 \AA$ and $2.17 \AA$ for exo and endo, respectively. The densities at bond critical point are 0.0196 au and 0.0180 au for exo and endo, respectively attesting to the presence of a bond path in both attacks. It has to be acknowledged that in both RPs the overall structure of the enols are nearly superimposable for the two attacks, their rmsd being $0.3 \AA$ and $0.1 \AA$ for PreRC1 and PreRC2, respectively.

Transition State (TS). For both reaction paths considered, the transition state leading to the exo product is favored (by $2.4 \mathrm{kcal}_{\mathrm{mol}}{ }^{-1}$ for TS1 and by $0.8 \mathrm{kcal} . \mathrm{mol}^{-1}$ for TS2), although (i) the $\mathrm{C}_{M}-\mathrm{C}_{3}$ distances are shorter for the two endo attacks and (ii) the positions of the incoming methyl chloride are only slightly nonsymmetrical for both attacks. In TS1, the $\mathrm{C}_{\mathrm{M}}-\mathrm{C}_{3}$ distances are 2.38 and $2.37 \AA$ for the exo and endo approaches, respectively. The endo attack leads to a less eclipsed position of the incoming methyl chloride $\left(\mathrm{C}_{\mathrm{M}}-\mathrm{C}_{3}-\mathrm{C}_{2}-\mathrm{O}\right.$ dihedral angle of $77^{\circ}$ and $75^{\circ}$, for exo and endo, respectively) because of sterical hindrances with the axial hydrogen of $\mathrm{C}_{6}$ and the alcohol function involved in $\mathrm{HB}-1$, resulting in a weaker $\mathrm{C}-\mathrm{H} \cdots \mathrm{O}$ interaction ( $2.93 \AA$ vs. $2.76 \AA$ for exo), a deformation of the piperazine cycle and a spatial rearrangement of HB-1, hence a destabilization. Indeed, the formation of the endo TS is impeded by the presence of the axial hydrogen of $\mathrm{C}_{6}$, with a $\mathrm{H}^{\cdots} \mathrm{H}$ distance of $2.33 \AA$, which is smaller than two times the van der Waals radius of hydrogen $\left(\mathrm{r}_{\mathrm{H}}=1.2 \AA\right)$. In TS2, by construction, only the steric hindrance with the axial hydrogen of $\mathrm{C}_{6}$ might remain. While the methyl chloride is less attracted by the 2-oxopiperazine in the HB-2 conformation (the $\mathrm{C}_{\mathrm{M}}-\mathrm{C}_{3}$ and $\mathrm{C}-\mathrm{H} \cdots \mathrm{O}$ distances are longer than in the HB-1 structures), there is no relevant difference in its location between the two attacks as there is no specific steric interaction in the TS2 transitions structures. The main difference between the two structures is the value of the $\mathrm{O}-\mathrm{C}_{2}-\mathrm{C}_{3}-\mathrm{H}$ dihedral angle, which is $25^{\circ}$ and $20^{\circ}$ for the exo and endo attacks respectively, attesting to slight non-equivalent torsional strains in the piperazine cycle upon alkylation at the $\mathrm{C}_{3}$ position. Consequently, the exo and endo TS2 are less than 1 kcal.mol ${ }^{-1}$ apart. The exo and endo structures of the "TS enol" show different deformations as confirmed by 
a rmsd of $0.56 \AA$ and $0.25 \AA$, respectively. It has to be acknowledged that the energy difference between the exo structures of TS1 and TS2 is of $2.9 \mathrm{kcal}^{\mathrm{mol}}{ }^{-1}$ in favor of TS1 in the gas phase.

Post-Reaction Complexes (PostRC). For both hydrogen patterns the product obtained through the exo attack is clearly favored and is around $5 \mathrm{kcal}^{\mathrm{mol}}{ }^{-1}$ more stable $\left(5.0 \mathrm{kcal} . \mathrm{mol}^{-1}\right.$ for HB-1 and $5.6 \mathrm{kcal}^{\mathrm{mol}}{ }^{-1}$ for HB2, see Tables 2 and 3). The exo attacks lead to (1'R,3S)-2 products with an equatorial $-\mathrm{CH}_{3}$ at the $\mathrm{C}_{3}$ position, while endo attacks lead to $\left(1^{\prime} R, 3 R\right)-2$ products with an axial $-\mathrm{CH}_{3}$ at the $\mathrm{C}_{3}$ position, inducing an additional strain/deformation of the piperazine cycle in this latter case. The methyl groups preferring the equatorial position, it becomes obvious that the exo products are favored. Upon alkylation, because of sterical hindrance, the Boc moiety is reoriented anti to the newly bound methyl, again inducing strain in the piperazine cycle. The released chloride anion interacts with two hydrogen atoms from the 2-oxopiperazine as a consequence of stabilizing electrostatic interactions. The overall enol structures (the newly bound $-\mathrm{CH} 3$ has not been considered) are now very different upon alkylation, as the rmsd between exo and endo configurations are now $1.36 \AA$ and $1.15 \AA$ for PostRC1 and PostRC2, respectively. It has to be acknowledged that the energy difference between the exo structures of PostRC1 and PostRC2 is 2.6 kcal.mol-1 in favor of PostRC1 in the gas phase.

The most relevant effect of the solvation is the stabilization of both the transition states and the post-reaction complexes, and in this latter case, the stabilization increases with the polarity of the solvent (Tables 2 and 3) because of a favorable solvation of the released chloride anion. The solvation does not change the exo/endo preferences but one can observe, especially for RP1, that the energy difference between the two enantiomers diminishes.

In RP1, from PreRC1 to TS1, there is a weakening of the HB-1 intramolecular hydrogen bond as its length increases from $1.75 \AA$ to $1.82 \AA$ in both attacks. As can be seen on Figures 3 and SI3-5, the spacial arrangement of HB-1 impedes the incoming of the methyl chloride in the endo attack. In order to keep the intramolecular hydrogen bond distance at its optimal value while favoring the ingress of the methyl group, the system has to deform (bending/rotation of the alcohol function). The slightly higher deformation of the overall enol structure upon incoming of the endo $\mathrm{MeCl}$ is confirmed by the evaluation of the rmsd which is of $0.49 \AA$ vs. 0.34 for the exo attack. Then from TS1 to PostRC1, the HB weakens again and now has a value of 1.93 and 1.90 for exo and endo products, respectively. Again, the overall deformation of the enol is more important for the endo configuration $(0.99 \AA$ vs. $0.84 \AA)$. The strong intramolecular hydrogen bonds in PreRC1 and TS1 locks the piperazine ring in its conformation, forcing its surroundings to deform and to adapt to the incoming methyl chloride. Upon alkylation and thus weakening of HB-1 because of the loss of the negative charge and subsequent equilibration of the electronic density, there is a release of the strain and the piperazine ring is able to deform in PostRC1. In RP2, compared to RP1, the intramolecular hydrogen bond is weaker by construction and its value does not vary much from PreRC2 to PostRC2 (see Figures 4 and SI6-8), attesting of its non-participation in the alkylation process. Deformations of the enol structure are also less important than in the HB-1 case. 
On the one hand, enols from 2-oxopiperazines featuring the intramolecular hydrogen bond pattern HB-1 are the most stable in the gas phase, but:

(i) upon alkylation, they show more structural deformation, as HB-1 has to adapt to local sterical hindrance and a change of the electronic density on the carbonyl function along the reaction pathway;

(ii) in solution, the exo/endo preference is not as marked as in the gas phase.

On the other hand, enols from 2-oxopiperazines featuring the intramolecular hydrogen bond pattern HB-2 are less prone to form in the gas phase, but

(i) upon alkylation, the exo/endo preference is more significant than in the HB-1 case, especially when solvent is involved;

(ii) there is little deformation of the overall piperazine moiety throughout the reaction path.

As aforementioned, the most stable conformation for the 2-oxopiperazine reactant $\left(1^{\prime} R\right)-1$ possesses an intramolecular HB-2 pattern, so it could be possible for the system to swap from one pattern to another in order to minimize the energy losses upon reorganization/deformation.

In order to prove the validity of this initial model, we performed additional calculations using a lithium atom in place of the hydroxyl hydrogen for the pre-reaction, transition state and post-reaction structures in both the exo and endo approaches. We focused on the one conformation where the lithium bridges the two anionic oxygen atoms. Free energy differences obtained in the gas phase as well as in a solvent medium for the two reaction paths are given in Table 4. Detailed structures are given in Supplementary Information (Figures SI9-11). Again, consistent with experimental results, the calculations favor the formation of the exo product by $4.7 \mathrm{kcal}_{\mathrm{mol}}{ }^{-1}$. This energy difference is comparable to that obtained for RP1 and RP2.

Similarly, the geometrical results and observations for the structures featuring the lithium atom along the reaction path are comparable to those obtained for RP1, i.e. (i) the endo approach of the methyl chloride is impeded by the $\mathrm{O} \cdots \mathrm{Li} \cdots \mathrm{O}$ interaction and the axial hydrogen at position $\mathrm{C}_{6}$, (ii) the transition state leading to the exo product is favored (by $1.6 \mathrm{kcal}_{\mathrm{mol}}{ }^{-1}$ ) and (iii) deformations to accommodate the bonding of the methyl are observed. The main difference between this scheme and RP1 is the relative evolutions of the lengths of $\mathrm{OLi} \cdots \mathrm{O}$ and $\mathrm{OH} \cdots \mathrm{O}$. In the previous scheme, the $\mathrm{OH} \cdots \mathrm{O}$ distance increases from PreRC1 to PostRC1, whereas in the new scheme the $\mathrm{OLi} \cdots \mathrm{O}$ distance remains constant. Indeed, the predominantly ionic nature of the $\mathrm{O} \cdots \mathrm{Li} \cdots \mathrm{O}$ "bonds" allows a larger conformational freedom to the lithium ion between the two anionic sites. The consequences of these looser bonds are a less pronounced exo/endo preference

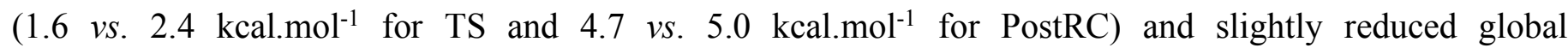
deformations. Upon solvation, the exo preference is kept, when focusing on the PostRC-Li figures of Table 4 only. As a reminder, the frequencies obtained in the RP-Li scheme the free energies computed in a solvent phase are obtained via $a h$ hoc corrections applied to a gas phase model, which is a valid approximation, since vibrational contributions to free energies are insensitive to whether they are computed from a gas 
phase structure or from a solvated one.[48] Contrary to the RP1 scheme, the endo approach of the methyl in the transition state structures is favored for the most polar and protic solvents, i.e. THF and water in our case. In these latter solvents, the endo TS is stabilized by a higher vibrational entropy contribution. This inversion of trend can arise from a combination of many factors: (i) a slightly different position of the methyl chloride in the exo and endo transition state structures induces a different exposition to the solvent for the methyl and $\mathrm{Cl}^{-}$moieties, (ii) the frequency calculations performed on the exo and endo TS structure do not necessarily provide a unique and equal number of imaginary frequencies, (iii) compared to the RP1 scheme, there are more "low-frequency" modes and (iv) the solvation of the Li cation might differ in the exo and endo TS structures. TS-Li, in the presence of THF or water, is a system particularly showing the limitations of the methodology, featuring both low-frequency vibrations and ions.[49] But one has to acknowledge that the exo preference is kept along the reaction path when the entropic corrections are not included in the calculation (see Tables SI1-3).

These additional calculations show that $-\mathrm{OLi}$ and $-\mathrm{OH}$ interact quite similarly with the nearby carbonyl function, which possesses a high electronic density. They provide validation of our simplified model and its a priori non-realistic reactive species (the non-deprotonated hydroxyl function). Modeling the reactant using a hydroxyl function and basing the discussion on hydrogen bonding patterns seems a relevant first approximation: this approach represents a good compromise between feasibility/limitations of the computational methods and the actual reaction occurring in the test tube; in particular, it avoids having to take into account aggregates formed by the lithium with its direct environment (THF solvent molecules) and prevents difficulties linked with the localization of the charge during the conformational search). The role of the chiral auxiliary, which was seen to induce rigidity and sterical hindrance via $-\mathrm{OH} /-\mathrm{OLi}$ interactions, yields a first insight into one of the many complex mechanisms underlying this diastereoselective alkylation.

\section{Conclusion:}

The computational results given in Table 1 unambiguously indicate a stereospecificity upon alkylation at position 3 of 2-oxopiperazines. Starting from a $1^{\prime} R$ configuration, the resulting $\left(1^{\prime} R, 3 S\right)$ is always favored over the $\left(1^{\prime} R, 3 R\right)$ one, which is totally consistent with experimental results. The free energy difference between the most stable $\left(1^{\prime} R, 3 S\right)$ and $\left(1^{\prime} R, 3 R\right)$ structures is $1.8 \mathrm{kcal} \cdot \mathrm{mol}^{-1}$ at a temperature of $-78^{\circ} \mathrm{C}$ and a pressure of $1 \mathrm{~atm}$, which corresponds to the experimental 98:2 ratio. From this result alone, the alkylation of 2-oxopiperazines would thus appear to be a thermodynamically driven reaction. However, the reaction paths leading to either the endo or the exo products and the energetics of the pre-reaction, post-reaction and transition state structures (Figure 3) unambiguously prove that the alkylation of 2-oxopiperazines is a kinetically driven reaction. Even when considering distinct conformations of the reactants, the exo attack is always favored over the endo one, even for the least stable of the TS conformers. The selectivity does not depend on the conformation of the starting enol reactant, but the mechanisms driving this selectivity might 
differ with the conformation. Indeed, the presence of the Boc moiety and the alcohol chiral auxiliary blocks the conformation of the piperazine cycle thus impeding a ring inversion and subsequently favoring an approach of the electrophile where the methyl will be equatorially bound. Moreover, the presence of an intramolecular hydrogen bond, i.e. the formation of a pseudo 7-ring, between the alcohol function of the chiral auxiliary and the carbonyl function of the piperazine adds a new level of rigidity to the 2oxopiperazine enol as well as sterical hindrance upon incoming of the methyl chloride, favoring the approach of the electrophile anti to the intramolecular hydrogen bond. These results confirm the early hypotheses of Micouin et al. on the origins of diastereoselectivity in the alkylation of $N$-substituted lactams.[38]

In conclusion, apart from sterical hindrance in some selected conformations, there is no special effect of the substituent, as the $\mathrm{C}_{3}-\mathrm{C}_{M}$ distance does not govern the stereoselectivity. The main source of the exo specificity upon alkylation is the imposed and controlled strain/rigidity of the piperazine ring imposed by the chiral auxiliary in the transition structure. Indeed, the preference for one conformation of this ring induces an enantiofacial selectivity upon approach of the electrophile.

Acknowledgements

The calculations presented in this work were performed using resources from the IDRIS facility (grant i2013087159) and the MeCS computing platform of Université de Picardie Jules Verne.

Bibliography

[1] J.P. Vacca, B.D. Dorsey, W.A. Schleif, R.B. Levin, S.L. McDaniel, P.L. Darke, J. Zugay, J.C. Quintero, O.M. Blahy, E. Roth, L-735,524: an orally bioavailable human immunodeficiency virus type 1 protease inhibitor, Proc. Natl. Acad. Sci. U.S.A., 91 (1994) 4096-4100.

[2] R. Capdeville, E. Buchdunger, J. Zimmermann, A. Matter, Glivec (STI571, imatinib), a rationally developed, targeted anticancer drug, Nat. Rev. Drug Discov., 1 (2002) 493-502.

[3] S.E. Ward, P. Eddershaw, S.T. Flynn, L. Gordon, P.J. Lovell, S.H. Moore, C.M. Scott, P.W. Smith, K.M. Thewlis, P.A. Wyman, Studies on a series of potent, orally bioavailable, 5-HT(1) receptor ligands--part II, Bioorg. Med. Chem. Lett., 19 (2009) 428-432.

[4] E.R. Ashley, E.G. Cruz, B.M. Stoltz, The total synthesis of (--)-lemonomycin, J. Am. Chem. Soc., 125 (2003) 15000-15001.

[5] M.L. Amador, J. Jimeno, L. Paz-Ares, H. Cortes-Funes, M. Hidalgo, Progress in the development and acquisition of anticancer agents from marine sources, Ann. Oncol., 14 (2003) 1607-1615. 
[6] S. Nagumo, A. Matoba, Y. Ishii, S. Yamaguchi, N. Akutsu, H. Nishijima, A. Nishida, N. Kawahara, Synthesis of (-)-TAN1251A using 4-hydroxy-1-proline as a chiral source, Tetrahedron, 58 (2002) 98719877.

[7] N.K. Garg, B.M. Stoltz, The formal total synthesis of dragmacidin B, trans-dragmacidin C, and cis- and trans-dihydrohamacanthins A, Tetrahedron Lett., 46 (2005) 2423-2426.

[8] H.J. Kim, W.Y. Kwak, J.P. Min, J.Y. Lee, T.H. Yoon, H.D. Kim, C.Y. Shin, M.K. Kim, S.H. Choi, H.S. Kim, E.K. Yang, Y.H. Cheong, Y.N. Chae, K.J. Park, J.M. Jang, S.J. Choi, M.H. Son, S.H. Kim, M. Yoo, B.J. Lee, Discovery of DA-1229: a potent, long acting dipeptidyl peptidase-4 inhibitor for the treatment of type 2 diabetes, Bioorg. Med. Chem. Lett., 21 (2011) 3809-3812.

[9] A. Khalaj, N. Adibpour, A.R. Shahverdi, M. Daneshtalab, Synthesis and antibacterial activity of 2-(4substituted phenyl)-3(2H)-isothiazolones, European journal of medicinal chemistry, 39 (2004) 699-705.

[10] M.D. Arbo, M.L. Bastos, H.F. Carmo, Piperazine compounds as drugs of abuse, Drug Alcohol Depend., 122 (2012) 174-185.

[11] P. Kukula, R. Prins, Diastereoselective Hydrogenation of Pyrazine Derivatives: An Alternative Method of Preparing Piperazine-(2S)-Carboxylic Acid, J. Catal., 208 (2002) 404-411.

[12] Z. Chen, A.S. Kende, A.O. Colson, J.L. Mendezandino, F.H. Ebetino, R.D. Bush, X.E. Hu, Ketopiperazines: Conformationally Constrained Peptidomimetic of Arginine Amides, Synth. Commun., 36 (2006) 473-479.

[13] A. Viso, R.F. de la Pradilla, A. Flores, A. García, M. Tortosa, M.L. López-Rodríguez, Synthesis of Highly Substituted Enantiopure Piperazines and Ketopiperazines from Vicinal N-Sulfinyl Diamines, The Journal of Organic Chemistry, 71 (2006) 1442-1448.

[14] G. Reginato, B. Di Credico, D. Andreotti, A. Mingardi, A. Paio, D. Donati, A new versatile and diastereoselective synthesis of polysubstituted 2-oxopiperazines from naturally occurring amino acids, Tetrahedron: Asymmetry, 18 (2007) 2680-2688.

[15] A. Pohlmann, V. Schanen, D. Guillaume, J.-C. Quirion, H.-P. Husson, Efficient Synthesis of Conformationally Constrained Peptidomimetics Containing 2-Oxopiperazines1, The Journal of Organic Chemistry, 62 (1997) 1016-1022.

[16] V. Schanen, C. Riche, A. Chiaroni, J.-C. Quirion, H.-P. Husson, Asymmetric synthesis. XXXI. Synthesis of 2-substituted piperazines from chiral non-racemic lactams, Tetrahedron Lett., 35 (1994) 25332536.

[17] V. Schanen, M.-P. Cherrier, S.J. Melo, J.-C. Quirion, H.-P. Husson, Asymmetric Synthesis; XXXVII: Synthesis of 2,6-Disubstituted Piperazines from Chiral Non-Racemic Lactams, Synthesis, 7 (1996).

[18] D. Hoppe, T. Hense, Enantioselective Synthesis with Lithium/(-)-Sparteine Carbanion Pairs, Angew. Chem. Int. Ed. Engl., 36 (1997) 2282-2316.

[19] B.P. McDermott, A.D. Campbell, A. Ertan, First Example of s-BuLi/(-)-Sparteine-Mediated Chiral Deprotonation of a Piperazine and Proof of the Sense of Induction, Synlett, 6 (2008) 875-879. 
[20] V. Santes, E. Gómez, V. Zárate, R. Santillan, N. Farfán, S. Rojas-Lima, Synthesis of new homochiral 2,3-dialkylpiperazines derived from (R)-(-)-phenylglycinol, Tetrahedron: Asymmetry, 12 (2001) 241-247.

[21] J. Seibel, D. Brown, A. Amour, S.J. Macdonald, N.J. Oldham, C.J. Schofield, Synthesis and evaluation of $\delta$-Lactams (Piperazones) as elastase inhibitors, Bioorg. Med. Chem. Lett., 13 (2003) 387-389.

[22] J. DiMaio, B. Belleau, Synthesis of chiral piperazin-2-ones as model peptidomimetics, J. Chem. Soc., Perkin Trans. 1, (1989) 1687-1689.

[23] G.P. Pollini, N. Baricordi, S. Benetti, C. De Risi, V. Zanirato, A simple entry to chiral non-racemic 2piperazinone derivatives, Tetrahedron Lett., 46 (2005) 3699-3701.

[24] C. De Risi, M. Pelà, G.P. Pollini, C. Trapella, V. Zanirato, Mastering chiral substituted 2oxopiperazines, Tetrahedron: Asymmetry, 21 (2010) 255-274.

[25] D.C. Beshore, C.J. Dinsmore, Preparation of substituted piperazinones via tandem reductive amination(N,N'-acyl transfer)-cyclization, Org. Lett., 4 (2002) 1201-1204.

[26] T. Prisinzano, K.C. Rice, M.H. Baumann, R.B. Rothman, Development of Neurochemical Normalization ("Agonist Substitution") Therapeutics for Stimulant Abuse: Focus on the Dopamine Uptake Inhibitor, GBR12909, Curr. Med. Chem.: Central Nervous System Agents, 4 (2004) 47-59.

[27] A. Pohlmann, D. Guillaume, J.-C. Quirion, H.-P. Husson, Synthesis and conformational analysis of two 2-oxopiperazine-containing tetrapeptide analogues, J. Pept. Res. , 51 (1998) 116-120.

[28] H. Kim, M.S. So, J. Chin, Preparation of Chiral Diamines by the Diaza-Cope Rearrangement (DCR), Aldrichimica Arta, 41 (2008) 77-88.

[29] C.J. Carrano, K.N. Raymond, Coordination chemistry of microbial iron transport compounds. 10. Characterization of the complexes of rhodotorulic acid, a dihydroxamate siderophore, J. Am. Chem. Soc., 100 (1978) 5371-5374.

[30] G. Muller, B.F. Matzanke, K.N. Raymond, Iron transport in Streptomyces pilosus mediated by ferrichrome siderophores, rhodotorulic acid, and enantio-rhodotorulic acid, J. Bacteriol., 160 (1984) 313318.

[31] B.F. Matzanke, G.I. Muller, K.N. Raymond, Hydroxamate siderophore mediated iron uptake in E. coli: stereospecific recognition of ferric rhodotorulic acid, Biochem. Biophys. Res. Commun., 121 (1984) 922930.

[32] N. Franceschini, P. Sonnet, D. Guillaume, Simple, versatile and highly diastereoselective synthesis of 1,3,4-trisubstituted-2-oxopiperazine-containing peptidomimetic precursors, Org. Biomol. Chem., 3 (2005) 787-793.

[33] C. Leoneti Lencina, A. Dassonville-Klimpt, P. Sonnet, New efficient enantioselective synthesis of 2oxopiperazines: a practical access to chiral 3-substituted 2-oxopiperazines, Tetrahedron: Asymmetry, 19 (2008) 1689-1697. 
[34] J.M. Um, N.S. Kaka, D.M. Hodgson, K.N. Houk, Transition states and origins of 1,4-asymmetric induction in alkylations of 2,2,6-trialkylpiperidine enamines, Chemistry A European Journal, 16 (2010) 6310.

[35] H.K. Khartabil, P.C. Gros, Y. Fort, M.F. Ruiz-López, A Theoretical Study on nBuLi/Lithium Aminoalkoxide Aggregation in Hexane and THF, The Journal of Organic Chemistry, 73 (2008) 9393-9402.

[36] W.F. Bailey, P. Beak, S.T. Kerrick, S. Ma, K.B. Wiberg, An experimental and computational investigation of the enantioselective deprotonation of Boc-piperidine, J. Am. Chem. Soc., 124 (2002) 18891896.

[37] I. Soteras, O. Lozano, A. Gomez-Esque, C. Escolano, M. Orozco, M. Amat, J. Bosch, F.J. Luque, On the origin of the stereoselectivity in the alkylation of oxazolopiperidone enolates, J. Am. Chem. Soc., 128 (2006) 6581-6588.

[38] L. Micouin, V. Jullian, J.-C. Quirion, H.-P. Husson, Origins of diastereoselectivity in the alkylation of $\mathrm{N}$-substituted lactams and amides derived from optically active aminoalcohols, Tetrahedron: Asymmetry, 7 (1996) 2839-2846.

[39] R. Brückner, Chemistry of the Alkaline Earth Metal Enolates in: H.A. Press (Ed.) Advanced Organic Chemistry: Reaction Mechanisms2002, pp. 373.

[40] H.J. Reich, Role of organolithium aggregates and mixed aggregates in organolithium mechanisms, Chem. Rev., 113 (2013) 7130-7178.

[41] Y. Ikuta, S. Tomoda, Origin of Stereochemical Reversal in Meyers-Type Enolate Alkylations. Importance of Intramolecular Li Coordination and Solvent Effects, Org. Lett., 6 (2004) 189-192.

[42] D. Romo, A.I. Meyers, Chiral non-racemic bicyclic lactams. Vehicles for the construction of natural and unnatural products containing quaternary carbon centers, Tetrahedron, 47 (1991) 9503-9569.

[43] M.J. Frisch, G.W. Trucks, H.B. Schlegel, G.E. Scuseria, M.A. Robb, J.R. Cheeseman, G. Scalmani, V. Barone, B. Mennucci, G.A. Petersson, H. Nakatsuji, M. Caricato, X. Li, H.P. Hratchian, A.F. Izmaylov, J. Bloino, G. Zheng, J.L. Sonnenberg, M. Hada, M. Ehara, K. Toyota, R. Fukuda, J. Hasegawa, M. Ishida, T. Nakajima, Y. Honda, O. Kitao, H. Nakai, T. Vreven, J.A. Montgomery Jr, J.E. Peralta, F. Ogliaro, M.J. Bearpark, J. Heyd, E.N. Brothers, K.N. Kudin, V.N. Staroverov, R. Kobayashi, J. Normand, K. Raghavachari, A.P. Rendell, J.C. Burant, S.S. Iyengar, J. Tomasi, M. Cossi, N. Rega, N.J. Millam, M. Klene, J.E. Knox, J.B. Cross, V. Bakken, C. Adamo, J. Jaramillo, R. Gomperts, R.E. Stratmann, O. Yazyev, A.J. Austin, R. Cammi, C. Pomelli, J.W. Ochterski, R.L. Martin, K. Morokuma, V.G. Zakrzewski, G.A. Voth, P. Salvador, J.J. Dannenberg, S. Dapprich, A.D. Daniels, Ö. Farkas, J.B. Foresman, J.V. Ortiz, J. Cioslowski, D.J. Fox, Gaussian 09, Revision D.01, Gaussian, Inc., Wallingford, CT, USA, 2009.

[44] A.D. Becke, Density - functional thermochemistry. III. The role of exact exchange, Journal of Chemical Physics, 98 (1993) 5648-5652.

[45] A.D. Becke, Density-functional exchange-energy approximation with correct asymptotic behavior, Physical review. A, 38 (1988) 3098-3100. 
[46] C. Lee, W. Yang, R.G. Parr, Development of the Colle-Salvetti correlation-energy formula into a functional of the electron density, Physical review. B, Condensed matter, 37 (1988) 785-789.

[47] T. Lu, F. Chen, Multiwfn: a multifunctional wavefunction analyzer, J. Comput. Chem., 33 (2012) 580592.

[48] R.F. Ribeiro, A.V. Marenich, C.J. Cramer, D.G. Truhlar, Use of solution-phase vibrational frequencies in continuum models for the free energy of solvation, J. Phys. Chem. B, 115 (2011) 14556-14562.

[49] J.H. Jensen, Predicting accurate absolute binding energies in aqueous solution: thermodynamic considerations for electronic structure methods, Phys. Chem. Chem. Phys., 17 (2015) 12441-12451. 
Table 1. Energy and free energy differences $\left(\mathrm{kcal}_{\mathrm{mol}}{ }^{-1}\right)$ of separated products computed at the B3LYP/6$31+\mathrm{G}^{*}$ and MP2 $/ 6-31+\mathrm{G}^{*}$ levels of theory at a temperature of $298 \mathrm{~K}$ and a pressure of $1 \mathrm{~atm} . \Delta \mathrm{E}$ energies are ZPE corrected.

\begin{tabular}{|c|c|c|c|c|c|c|c|}
\hline & \multicolumn{2}{|c|}{ Gas phase } & \multirow{2}{*}{$\frac{\mathrm{CCl}_{4}}{\Delta \mathrm{G}}$} & \multirow{2}{*}{$\begin{array}{c}\mathrm{CHCl}_{3} \\
\Delta \mathrm{G}\end{array}$} & \multirow{2}{*}{$\begin{array}{c}\text { THF } \\
\Delta \mathrm{G}\end{array}$} & \multirow{2}{*}{$\frac{\text { n-Octanol }}{\Delta \mathrm{G}}$} & \multirow{2}{*}{$\begin{array}{c}\text { Water } \\
\Delta \mathrm{G}\end{array}$} \\
\hline & $\Delta(\mathrm{E}+\mathrm{ZPE})$ & $\Delta \mathrm{G}$ & & & & & \\
\hline \multicolumn{8}{|c|}{ B3LYP/6-31+G* } \\
\hline 2 exo & -33.6 & -29.2 & -45.3 & -50.6 & -51.9 & -52.5 & -53.1 \\
\hline 3 endo & -31.5 & -27.3 & -43.9 & -48.8 & -50.3 & -51.0 & -51.7 \\
\hline$\Delta$ & 2.1 & 1.9 & 1.4 & 1.8 & 1.6 & 1.5 & 1.4 \\
\hline \multicolumn{8}{|c|}{$\mathrm{MP} 2 / 6-31+\mathrm{G}^{*}$} \\
\hline 2 exo & -39.8 & -33.4 & -55.9 & -50.5 & -57.4 & -58.0 & -59.4 \\
\hline 3 endo & -37.5 & -31.6 & -54.0 & -48.7 & -55.5 & -56.1 & -57.4 \\
\hline$\Delta$ & 2.3 & 1.8 & 1.9 & 1.8 & 1.9 & 1.9 & 2.0 \\
\hline
\end{tabular}


Table 2. Free energy differences (in kcal. $\mathrm{mol}^{-1}$ ) at a temperature of $195 \mathrm{~K}$ and a pressure of 1 atm of PreReaction complexes, Transition States and Post-Reaction complexes for RP1 in the gas phase and in solution.

\begin{tabular}{|l|l|c|c|c|}
\hline solvent & approach & Pre-RC & TS & Post-RC \\
\hline gas phase & exo & 0.0 & 10.3 & -45.0 \\
& endo & 1.4 & 12.7 & -40.0 \\
\hline $\mathrm{CCl}_{4}$ & exo & 0.0 & 7.4 & -49.7 \\
& endo & 0.2 & 9.4 & -46.1 \\
\hline $\mathrm{CHCl}_{3}$ & exo & 0.0 & 8.6 & -50.1 \\
& endo & 0.6 & 9.4 & -49.0 \\
\hline $\mathrm{THF}$ & exo & 0.0 & 8.5 & -50.4 \\
& endo & 0.8 & 9.0 & -49.8 \\
\hline \multirow{2}{*}{ n-Octanol } & exo & 0.0 & 8.5 & -50.5 \\
& endo & 0.9 & 8.7 & -50.3 \\
\hline \multirow{2}{*}{ Water } & exo & 0.1 & 7.5 & -52.0 \\
& endo & 0.0 & 8.5 & -51.5 \\
\hline
\end{tabular}

Table 3. Free energy differences (in kcal. $\mathrm{mol}^{-1}$ ) at a temperature of $195 \mathrm{~K}$ and a pressure of 1 atm of PreReaction complexes, Transition States and Post-Reaction complexes for RP2 in the gas phase and in solution.

\begin{tabular}{|l|l|c|c|c|}
\hline solvent & approach & Pre-RC & TS & Post-RC \\
\hline gas phase & exo & 0.0 & 7.6 & -53.2 \\
& endo & 0.0 & 8.4 & -47.6 \\
\hline $\mathrm{CCl}_{4}$ & exo & 0.0 & 5.1 & -56.1 \\
& endo & 0.7 & 5.7 & -52.9 \\
\hline $\mathrm{CHCl}_{3}$ & exo & 0.0 & 5.9 & -55.5 \\
& endo & 1.0 & 7.0 & -53.5 \\
\hline THF & exo & 0.0 & 7.2 & -55.2 \\
& endo & 1.2 & 7.1 & -53.5 \\
\hline n-Octanol & exo & 0.6 & 6.1 & -56.2 \\
& endo & 0.0 & 6.0 & -54.5 \\
\hline \multirow{2}{*}{ Water } & exo & 0.0 & 5.6 & -57.2 \\
& endo & 0.3 & 5.2 & -55.9 \\
\hline
\end{tabular}


Table 4. Free energy differences (in kcal.mol-1), at a temperature of $195 \mathrm{~K}$ and a pressure of 1 atm of PreReaction complexes, Transition States and Post-Reaction complexes for RP-Li in the gas phase and in solution.

\begin{tabular}{|l|l|c|c|c|}
\hline solvent & approach & Pre-RC & TS & Post-RC \\
\hline gas phase & exo & 0.0 & 12.1 & -38.6 \\
& endo & 1.3 & 13.7 & -33.9 \\
\hline $\mathrm{CCl}_{4}$ & exo & 0.0 & 8.9 & -45.7 \\
& endo & 1.5 & 10.4 & -42.4 \\
\hline $\mathrm{CHCl}_{3}$ & exo & 0.9 & 6.2 & -48.9 \\
& endo & 0.0 & 6.3 & -46.3 \\
\hline THF & exo & 0.0 & 6.1 & -50.9 \\
& endo & 0.4 & 5.2 & -49.4 \\
\hline \multirow{2}{*}{ n-Octanol } & exo & 0.4 & 5.5 & -51.8 \\
& endo & 0.0 & 7.0 & -50.2 \\
\hline \multirow{2}{*}{ Water } & exo & 0.3 & 6.7 & -52.2 \\
& endo & 0.0 & 6.0 & -51.8 \\
\hline
\end{tabular}


Scheme 1. Alkylation of 2-oxopiperazine (1'R)-1 and (1'S)-1.

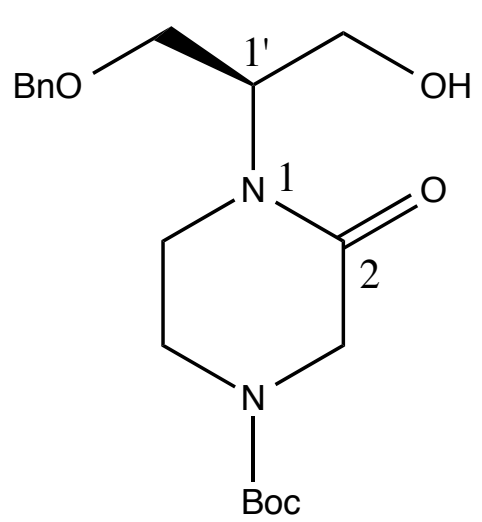

$(1 ' R)-1$

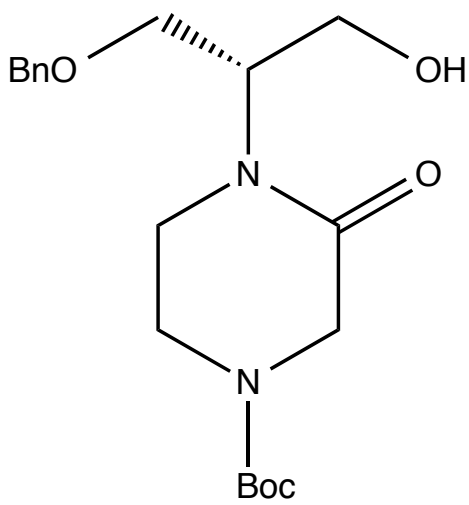

(1'S)-1
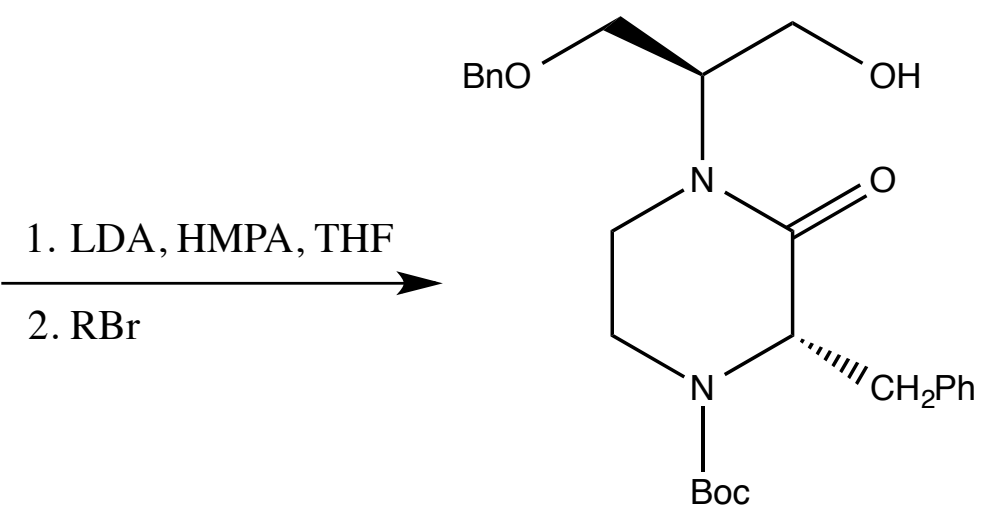

(1'R,3S)-2
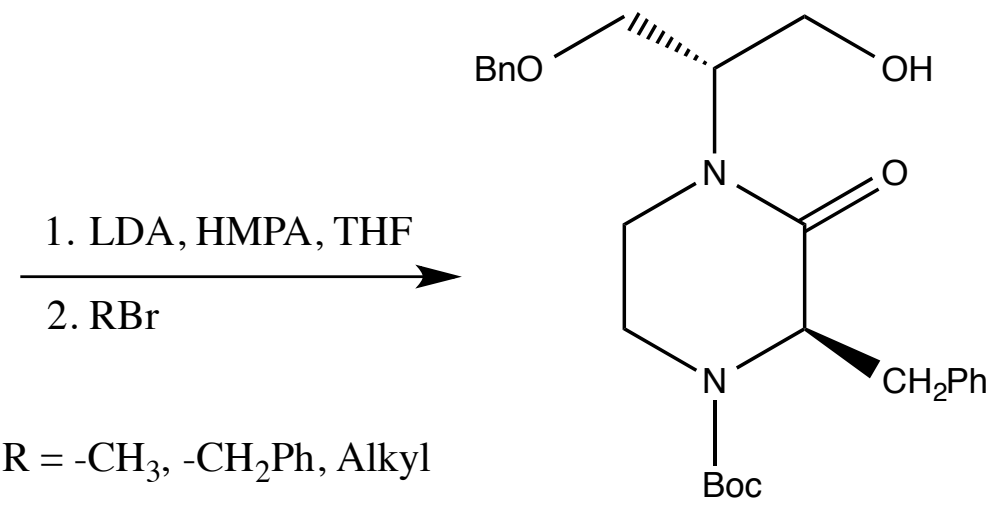

$(1 ' S, 3 R)-2$ 
Scheme 2.

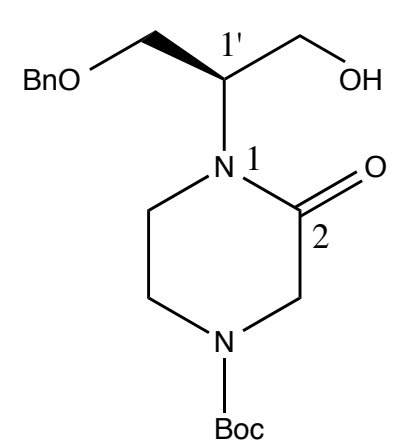

(1'R)-1

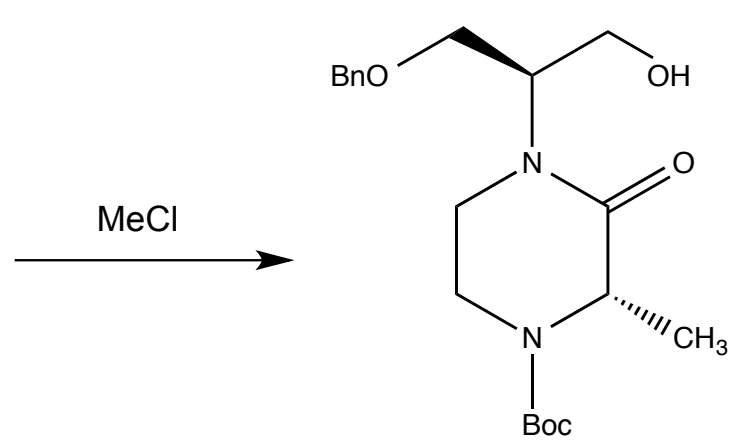

(1'R,3S)-2

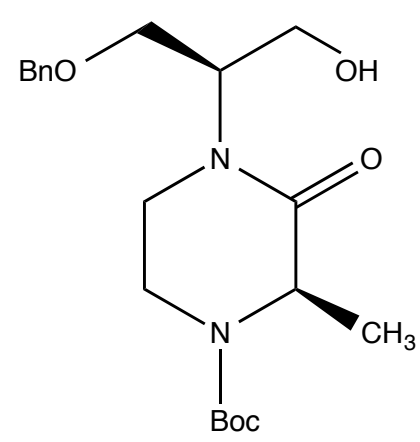

$\left(1^{\prime} R, 3 R\right)-2$ 
Figure 1. Structure of a piperazine (a), a 2-oxopiperazine (b) and Rhodotorulic acid (c).

(a)<smiles>C1CNCCN1</smiles>

(b)<smiles>O=C1CNCCN1</smiles>

(c)<smiles>CC(=O)N(O)CCC[C@@H]1NC(=O)[C@@H](CCCN(O)C(C)=O)NC1=O</smiles>

Figure 2. Intramolecular hydrogen bonding patterns. (a) HB-1 and (b) HB-2.

(a)<smiles>[R]C1C(=O)N([C@H](CO)COCc2ccccc2)CCN1C(=O)OC(C)(C)C</smiles>

(b)<smiles>CCCC1C(=O)N([C@@H]2CO[C@H](c3ccccc3)C2)CCN1C(=O)OC(C)(C)C</smiles> 


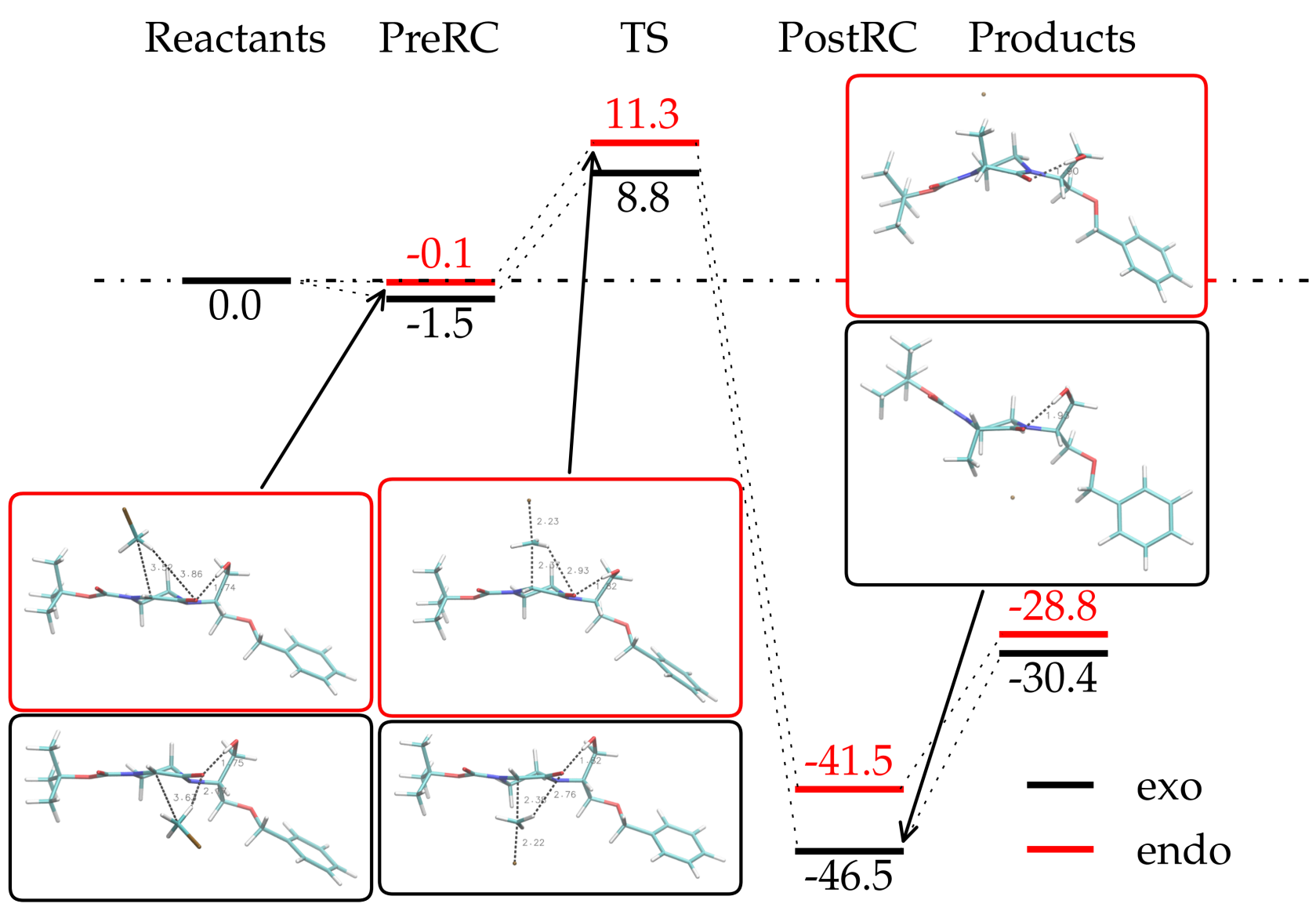

Figure 3. Pre-reaction (PreRC), transition state (TS) and post-reaction (PostRC) structures for the exo and endo attacks of the HB-1 intramolecular hydrogen-bonded enolate of (1'R)-1 by methyl chloride. The free energy differences between each state and the reactants are given in kcal.mol ${ }^{-1}$. Better quality images of the structures are given in Supplementary Information (SI3-5). 


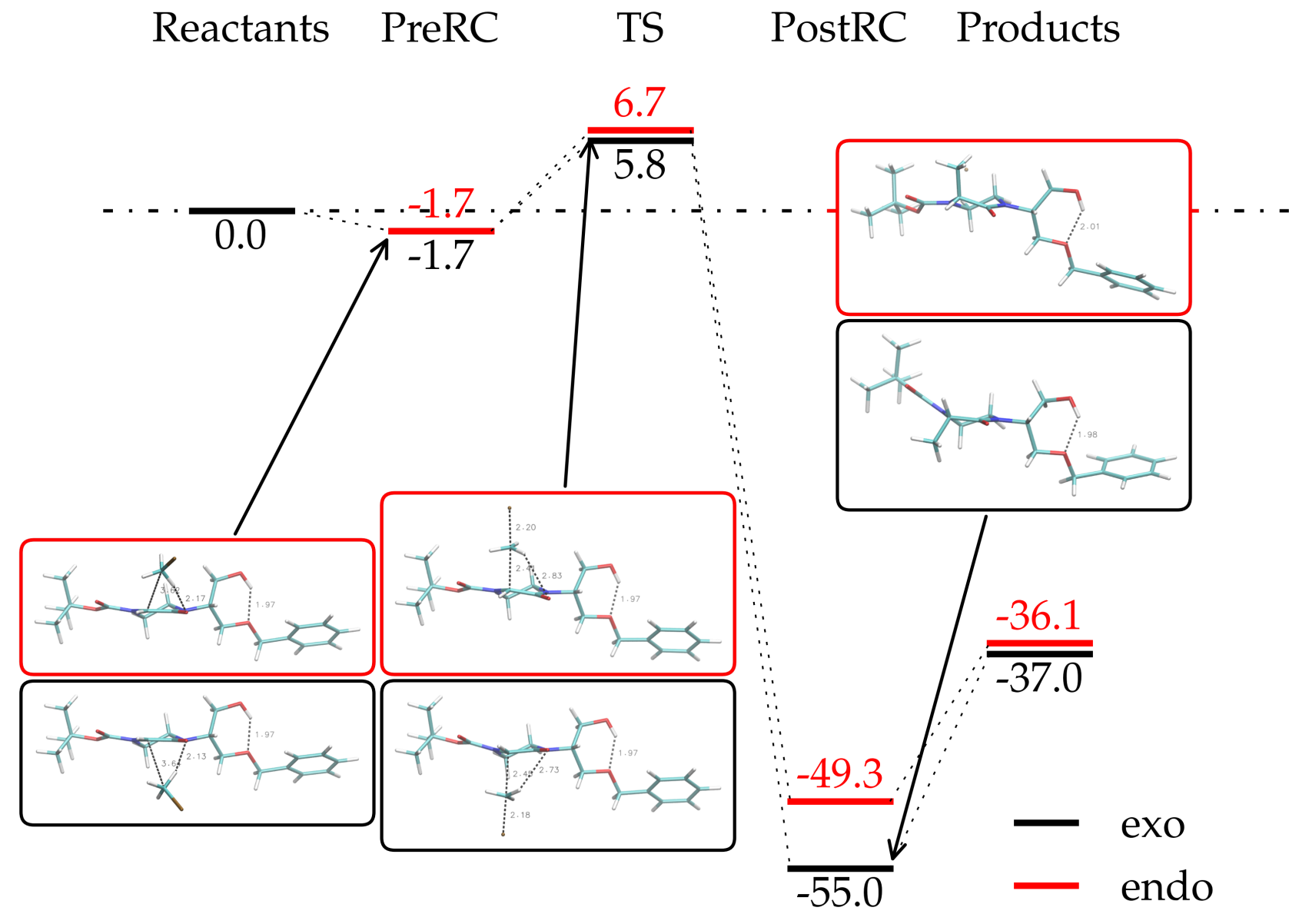

Figure 4. Pre-reaction (PreRC), transition state (TS) and post-reaction (PostRC) structures for the exo and endo attacks of the HB-2 intramolecular hydrogen-bonded enolate of (1'R)-1 by methyl chloride. The free energy differences between each state and the reactants are given in kcal.mol ${ }^{-1}$. Better quality images of the structures are given in Supplementary Information (SI6-9). 